\title{
EL REFORZAMIENTO ACADÉMICO EN EL APRENDIZAJE DE LA MATEMÁTICA Y SU RELACIÓN CON EL RENDIMIENTO ACADÉMICO EN ESTUDIANTES DE INGENIERÍA DE LA UNJBG DE TACNA EN EL AÑO 2016.
}

\author{
THE REINFORCEMENT IN THE MATH LEARNING AND ITS RELATION WITH THE LEVEL OF \\ ACADEMIC PERFORMANCE IN THE ENGINEER STUDENTS OF THE UNJBG OF TACNA IN THE \\ YEAR 2016
}

\author{
Julia Marina Mendoza Gómes', . Dionicio Milton Chávez Muñoz ${ }^{2}$
}

\section{RESUMEN}

La presente investigación se propuso determinar la relación que existe entre el reforzamiento académico en el aprendizaje de la matemática y el rendimiento académico en estudiantes de ingeniería de la UNJBG de Tacna en el año 2016. Precisar el nivel de reforzamiento académico en el aprendizaje de la matemática que presentan los estudiantes de ingeniería. Precisar el nivel de rendimiento académico que presentan los estudiantes de ingeniería en la asignatura de matemática. Se ha realizado una investigación de tipo no experimental, relacional que incluye lo descriptivo y explicativo, con un diseño de investigación transversal y con aplicación de encuestas a un total de 225 estudiantes.

Las siguientes conclusiones son:

Los estudiantes de las escuelas de ingeniería presentan un buen nivel en el reforzamiento académico de su aprendizaje en matemática, tendiendo al nivel de regular en la asignatura de matemática.

El rendimiento académico de los estudiantes de las escuelas de ingeniería se explica por su relación con el reforzamiento académico del aprendizaje de matemática del estudiante en el orden del 42,1\%. La relación corresponde a directa y buena.

PALABRAS CLAVE: reforzamiento académico en el aprendizaje, rendimiento académico.

\section{ABSTRACT}

The present research work was aimed to stablish the relation that exist between the academic reinforcement in the math learning and the academic performance in the engineer students of the UNJBG in the year 2016. To stablish the level of academic reinforcement in the math learning that the engineer students show. To stablish the level of academic perormance that the engineer students show in the subject of math. A non-experimental and relational research has been done, it includes the describing part and the explaining part, with a transversal research design and the aplying of 225 polls for students. These conclusions were came to:

The engineer schools students show a good level in the academic reinforcement of their math learning with a medium level trend in the subject of math.

The academic performance of the engineer schools students is explained by its relation with the acadmic reinforcement of the math learning of the students in $42,1 \%$. The relation is considered as direct and good.

KEYWORDS: Academic reinforcement in the learning, academic performance.

1 Doctora en Educación con mención en Gestión Educativa

Docente de la Facultad de Ciencias - Universidad Nacional Jorge Basadre Grohmann - Tacna Perú

2 Doctor en Educación con mención en Gestión Educativa

Docente de la Facultad de Ciencias - Universidad Nacional Jorge Basadre Grohmann - Tacna Perú 


\section{INTRODUCCIÓN}

La formación académica de los estudiantes de una universidad tienen aciertos que conducen a una buena formación pero también limitaciones de diversa índole que contribuyen a tener problemas en la formación, entre ellas se advierten escaso uso de estrategias de aprendizaje y pocos estilos de estudio que le ayuden a comprender mejor asignaturas como por ejemplo la matemática; entonces el estudiante tiene la necesidad de reforzar su aprendizaje con miras a mejorar su rendimiento académico, este reforzamiento se puede desagregar en dimensiones de tipo contextual, motivacional, cognitivo en clase y fuera de clase, es decir estudiando con el apoyo de otros mediadores, usando nuevas estrategias, otras técnicas y otras fuentes de información, inclusive mejorando su ambiente de estudio; y en la medida que lo haga mejorarán su aprendizaje, su desempeño y su rendimiento académico.

El reforzamiento en la asignatura de matemática contribuye a que el estudiante comprenda mejor la enseñanza proporcionada por el docente, dado que las horas de clase son limitadas para abordar todos los temas y toda la gama de ejercicios y problemas que tiene cada tópico de matemática. Una buena orientación por parte del profesor o un acertado desempeño por parte del estudiante ayudarán en el aprendizaje.

Finalmente, esta investigación servirá como punto de partida para proponer a las escuelas profesionales planes y programas de mejora en el aprendizaje de los estudiantes de las escuelas de ingeniería de la UJNBG, así como también capacitación didáctica y metodologías de enseñanza por parte del estudiante.

\section{MATERIALES Y MÉTODO}

\section{TÉCNICAS E INSTRUMENTOS DE RECOLECCIÓN DE DATOS.}

La recopilación de la información exigió la aplicación de encuestas tanto para determinar las formas de reforzamiento del aprendizaje de los estudiantes, como para determinar el Rendimiento Académico que alcanzan los estudiantes. Luego se procesaron los datos estadísticamente y se realizó la interpretación y el análisis respectivo.

\section{RESULTADOS}

Los resultados que se presentan han sido realizados en los cuatro aspectos evaluados del Reforzamiento del aprendizaje de la Matemática de los estudiantes de ingeniería, considerados en la presente investigación: Motivación del estudiante en su carrera de ingeniería; actuación del estudiante en el aula de clases; a del estudiante fuera del aula de clases; ambiente de estudio del estudiante.

\section{RESULTADOS RESPETO AL SEXO DEL ESTUDIANTE}

TABLA N ${ }^{\circ}$ 1. Distribución de resultados respecto al sexo del estudiante en la carrera de Ingeniería.

\begin{tabular}{cccc}
\hline & \multicolumn{3}{c}{$\begin{array}{c}\text { Sexo de los estudiantes } \\
\text { en Ingeniería }\end{array}$} \\
\cline { 2 - 4 } & $\mathrm{n}$ & $\%$ & Total \\
Masculino & 173 & 76,9 & 173 \\
Femenino & 52 & 23,1 & 52 \\
\hline Total & 225 & 100 & 225
\end{tabular}

Fuente: Encuesta para estudiantes.

INTERPRETACIÓN. En la Tabla y Figura $N^{\circ} 1$ se observa que la mayor cantidad de estudiantes son varones con $76,9 \%$ y el $23,1 \%$ son mujeres.

\section{RESULTADOS DE LA MOTIVACIÓN DEL ESTUDIANTE}

TABLA N ${ }^{\circ}$ 2. Distribución de resultados respecto a la motivación del estudiante en la carrera de Ingeniería.

\begin{tabular}{lcccccc}
\hline \multicolumn{5}{c}{ Motivación del } \\
& \multicolumn{3}{c}{ estudiante en su carrera } \\
& $\mathrm{n}$ & $\%$ & $\mathrm{n}$ & $\%$ & $\mathrm{n}$ & $\%$ \\
\cline { 2 - 8 } Bajo & 0 & 0 & 0 & 0 & 0 & 0 \\
Regular & 15 & 8,7 & 8 & 15,4 & 23 & 10,2 \\
Bueno & 103 & 59,5 & 27 & 51,9 & 130 & 57,8 \\
Muy & 55 & 31,8 & 17 & 32,7 & 72 & 32,0 \\
bueno & 173 & 100 & 52 & 100 & 225 & 100
\end{tabular}

Fuente: Encuesta para estudiantes. 
INTERPRETACIóN. En la Tabla se observa que los estudiantes varones tienen en mayor medida una buena motivación del $59,5 \%$ y una muy buena motivación del 31,8 \%; mientras que las mujeres tienen en mayor medida una buena motivación del 51,9 \% y una muy buena motivación del 32,7\%.

En total los estudiantes tienen una buena motivación del 57,8 \% y una muy buena motivación del $32 \%$. Una regular motivación solo con el $10,2 \%$.

\section{RESULTADOS DE LA ACTUACIóN EN EL AULA.}

TABLA N ${ }^{\circ}$ 3. Distribución de resultados respecto a la Actuación en el Aula del estudiante en la carrera de Ingeniería.

\begin{tabular}{|c|c|c|c|c|c|c|}
\hline & \multicolumn{4}{|c|}{$\begin{array}{l}\text { Actuación en el aula } \\
\text { del estudiante de } \\
\text { Ingeniería }\end{array}$} & \multirow{2}{*}{\multicolumn{2}{|c|}{ Total }} \\
\hline & \multicolumn{2}{|c|}{ Masculino } & \multicolumn{2}{|c|}{ Femenino } & & \\
\hline & $\mathrm{n}$ & $\%$ & $n$ & $\%$ & \multicolumn{2}{|r|}{$\%$} \\
\hline Bajo & 1 & 0,6 & 0 & 0,0 & 1 & 0,4 \\
\hline Regular & 69 & 39,9 & 22 & 42,3 & 91 & 40,4 \\
\hline Bueno & 93 & 53,8 & 29 & 55,8 & 122 & 54,2 \\
\hline $\begin{array}{l}\text { Muy } \\
\text { bueno }\end{array}$ & 10 & 5,8 & 1 & 1,9 & 11 & 4,9 \\
\hline Total & 173 & 100 & 52 & 100 & 225 & 100 \\
\hline \multicolumn{7}{|c|}{ Fuente: Encuesta para estudiantes. } \\
\hline \multicolumn{7}{|c|}{$\begin{array}{l}\text { INTERPRETAClóN. En la Tabla } N^{\circ} 3 \text { se observa } \\
\text { que los estudiantes varones tienen en mayor } \\
\text { medida una buena actuación en el aula del } 53,8 \\
\% \text { y una regular actuación en el aula del } 39,9 \% \text {; } \\
\text { mientras que las mujeres tienen en mayor } \\
\text { medida una buena actuación en el aula del 55,8 } \\
\% \text { y una regular actuación en el aula del } 42,3 \% \text {. }\end{array}$} \\
\hline \multicolumn{7}{|c|}{$\begin{array}{l}\text { En total los estudiantes tienen una buena } \\
\text { actuación en el aula del } 54,2 \% \text { y una muy buena } \\
\text { actuación en el aula del } 40,4 \% \text {. Una regular } \\
\text { actuación en el aula del } 4,9 \% \text {. }\end{array}$} \\
\hline \multicolumn{7}{|c|}{$\begin{array}{l}\text { RESULTADOS } \\
\text { DEL AULA }\end{array}$} \\
\hline \multicolumn{7}{|c|}{$\begin{array}{l}\text { TABLA N }{ }^{\circ} 4 \text {. Distribución de resultados respecto } \\
\text { a la Actuación fuera del Aula del estudiante en } \\
\text { la carrera de Ingeniería. }\end{array}$} \\
\hline
\end{tabular}

\begin{tabular}{lcccccc}
\hline \multicolumn{7}{c}{ Actuación fuera del } \\
aula del estudiante de \\
Ingeniería \\
& $\mathrm{n}$ & $\%$ & $\mathrm{n}$ & $\%$ & $\mathrm{n}$ & $\%$ \\
\cline { 2 - 8 } Basculino & \multicolumn{1}{c}{ Femenino } & \multicolumn{2}{c}{ Total } \\
Regular & 98 & 56,6 & 30 & 57,7 & 128 & 56,9 \\
Bueno & 56 & 32,4 & 14 & 26,9 & 70 & 31,1 \\
Muy & 6 & 3,5 & 3 & 5,8 & 9 & 4,0 \\
bueno & 13 & 7,5 & 5 & 9,6 & 18 & 8,0 \\
\hline \multicolumn{1}{c}{ Total } & 173 & 100 & 52 & 100 & 225 & 100 \\
Fuente: Encuesta para estudiantes & &
\end{tabular}

INTERPRETAClóN. En la Tabla $N^{\circ} 4$ se observa que los estudiantes varones tienen en mayor medida una regular actuación fuera del aula del $56,6 \%$ y una buena actuación fuera del aula del $32,4 \%$; mientras que las mujeres tienen en mayor medida una regular actuación fuera del aula del $57,7 \%$ y una buena actuación fuera del aula del $26,9 \%$.

En total los estudiantes tienen una regular actuación fuera del aula del 56,9 \% y una buena actuación fuera del aula del 31,1 \%. Una baja actuación fuera del aula del $8 \%$.

\section{RESULTADOS RESPECTO AL AMBIENTE DE ESTUDIO}

TABLA N ${ }^{\circ}$ 5. Distribución de resultados respecto al Ambiente de estudio del estudiante en la carrera de Ingeniería.

\begin{tabular}{lcccccc}
\hline \multicolumn{7}{c}{ Ambiente de Estudio } \\
del estudiante de \\
Ingeniería
\end{tabular}

Fuente: Encuesta para estudiantes

INTERPRETAClóN. En la Tabla $N^{\circ} 5$ se observa que los estudiantes varones tienen en mayor medida un regular ambiente de estudio del 43,9 $\%$ y un buen ambiente de estudio del 39,9\%; 
mientras que las mujeres tienen en mayor medida un regular ambiente de estudio del 44,2 $\%$ y un buen ambiente de estudio del $38,5 \%$.

En total los estudiantes tienen un regular ambiente de estudio del $44 \%$ y un buen ambiente de estudio del 39,6 \%. Un bajo ambiente de estudio del $9,3 \%$.

\section{RESULTADOS ACUMULADOS RESPECTO AL REFORZAMIENTO EN EL APRENDIZAJE}

TABLA N ${ }^{\circ}$ 6. Distribución de resultados respecto al reforzamiento en el aprendizaje del estudiante en la carrera de Ingeniería.

\begin{tabular}{|c|c|c|c|c|c|c|}
\hline & \multicolumn{4}{|c|}{$\begin{array}{l}\text { Reforzamiento en el } \\
\text { aprendizaje del } \\
\text { estudiante }\end{array}$} & \multirow{2}{*}{\multicolumn{2}{|c|}{ Total }} \\
\hline & \multicolumn{2}{|c|}{ Masculino } & \multicolumn{2}{|c|}{ Femenino } & & \\
\hline & $\mathrm{n}$ & $\%$ & $\mathrm{n}$ & $\%$ & $\mathrm{n}$ & $\%$ \\
\hline Bajo & 0 & 0,0 & 0 & 0,0 & 0 & 0,0 \\
\hline Regular & 59 & 34,1 & 24 & 46,2 & 83 & 36,9 \\
\hline Bueno & 108 & 62,4 & 27 & 51,9 & 135 & 60,0 \\
\hline $\begin{array}{l}\text { Muy } \\
\text { bueno }\end{array}$ & 6 & 3,5 & 1 & 1,9 & 7 & 3,1 \\
\hline Total & 173 & 100 & 52 & 100 & 225 & 100 \\
\hline
\end{tabular}

INTERPRETACIÓN . En la Tabla N ${ }^{\circ} 6$ se observa que los estudiantes varones tienen en mayor medida un buen reforzamiento en el aprendizaje del $62,4 \%$ y un regular reforzamiento del $34,1 \%$; mientras que las mujeres tienen en mayor medida un buen reforzamiento en el aprendizaje del $51,9 \%$ y un regular reforzamiento del $46,2 \%$.

\section{RESULTADOS DE LA APLICACIóN DE INSTRUMENTOS PARA EVALUAR EL RENDIMIENTO ACADÉMICO.}

Los resultados que se presentan han sido realizados en los dos aspectos evaluados del Rendimiento Académico de los estudiantes, considerados en la investigación: La forma en que el estudiante es evaluado; el Rendimiento académico propiamente dicho que se consolidan a continuación.

\section{RESULTADOS ACUMULADOS RESPECTO AL RENDIMIENTO ACADÉMICO}

TABLA N ${ }^{\circ}$ 9. Distribución de resultados respecto al Rendimiento académico del estudiante en la carrera de Ingeniería.

\begin{tabular}{lcccccc}
\hline \multicolumn{5}{c}{$\begin{array}{l}\text { Rendimiento académico } \\
\text { del estudiante de } \\
\text { ingeniería } \\
\text { Masculino }\end{array}$} & Femenino & \multicolumn{2}{c}{ Total } \\
Bajo & $\mathrm{n}$ & $\%$ & $\mathrm{n}$ & $\%$ & $\mathrm{n}$ & $\%$ \\
\cline { 2 - 8 } & 0 & 0,0 & 0 & 0,0 & 0 & 0,0 \\
Regular & 32 & 18,5 & 15 & 28,8 & 47 & 20,9 \\
Bueno & 106 & 61,3 & 23 & 44,2 & 129 & 57,3 \\
Muy & 35 & 20,2 & 14 & 26,9 & 49 & 21,8 \\
bueno & 173 & 100 & 52 & 100 & 225 & 100 \\
\hline Total & 173 \\
Fuente: Encuesta para estudiantes. & &
\end{tabular}

INTERPRETACIÓN. En la Tabla N 9 se observa que los estudiantes varones tienen en mayor medida un buen rendimiento académico del 48,6 $\%$ y un regular rendimiento académico del 40,5 $\%$; mientras que las mujeres tienen en mayor medida un buen rendimiento académico del 48,1 $\%$ y un regular rendimiento académico del $50 \%$.

\section{DISCUSIÓN}

\section{EL REFORZAMIENTO ACADÉMICO EN EL APRENDIZAJE DE LA MATEMÁTICA}

\section{Respecto a la motivación}

Este indicador se particulariza estableciendo que la carrera que el estudiante ha elegido, colma sus expectativas, por lo que se esfuerza para lograrlo, consulta sobre la carrera, está siempre motivado y expresa que desde el colegio fue de regular a más en matemática.

\section{Respecto a la actuación en el aula}

Este indicador de la actuación el estudiante en el aula, se particulariza estableciendo que la carrera que el estudiante ha decidido estudiar requiere del uso de conocimientos anteriores, que el estudiante consulte al profesor sobre sus dudas, hay una acertada participación en clase, toma apuntes adecuadamente, los estudia, consulta y compartes con sus compañeros, no requiere de reiteración explicativa de los temas, plantea solución a problemas, va a la par con sus compañeros y poco se distrae en clases.

\section{Respecto a la actuación fuera de clase}

Para ello el estudiante se reúne con sus compañeros para estudiar, estudia sus apuntes, de ser necesario busca un profesor particular 
para absolver sus dudas, refuerza sus conocimientos con textos del internet, con videos; hace trabajos en grupo, es decir en sus tiempos libres estudia la asignatura de matemática.

\section{Respecto al ambiente de estudio}

En particular los datos muestran que el estudiante tiene un ambiente de estudio, se distrae poco, dispone un horario para las asignatura de matemática, tiene lo necesario para estudiar esta asignatura y que sus padres le consultan sobre su avance en esta asignatura.

\section{EL RENDIMIENTO ACADÉMICO.}

Respecto a las formas de ser evaluado

Este indicador se concreta teniendo en cuenta que el profesor les aplica exámenes parciales, que les califica las prácticas aplicadas en el aula, que les considera y revisa las prácticas y los trabajos encargados para complementar y reforzar su aprendizaje en la signatura de matemática.

\section{Respecto al rendimiento académico}

Este indicador se concreta mostrando que el estudiante muestra que su conocimiento en matemática es bueno, que es regular en el desarrollo de prácticas, regular respondiendo a las preguntas del profesor, bueno presentado trabajos y prácticas encargadas y que en general su desempeño en la asignatura de matemáticas es bueno.

\section{CONCLUSIONES}

PRIMERA. Los estudiantes de las escuelas de ingeniería presentan un buen nivel en el reforzamiento académico de su aprendizaje, tendiendo al nivel de regular en la asignatura de matemática.

SEGUNDA. Los estudiantes de las escuelas de ingeniería presentan un buen nivel en el rendimiento académico, con tendencia al nivel de regular en la asignatura de matemática.

TERCERA. La relación que existe entre el reforzamiento académico en el aprendizaje de la matemática y el rendimiento académico en estudiantes de las escuelas de ingeniería de la UNJBG de Tacna en el año 2016 es directa y significativa.
CUARTA. El rendimiento académico de los estudiantes de las escuelas de ingeniería se explica por el reforzamiento del aprendizaje de matemática del estudiante en el orden del $42,1 \%$. La relación corresponde a directa y buena.

\section{REFERENCIAS BIBLIOGRÁFICAS}

ALONSO, C.; GALLEGO, D.; HONEY, P. (1994). Los Estilos de Aprendizaje. Procedimientos de diagnóstico y Mejora (6a Edición). Bilbao: Ediciones Mensajero.

BRUNER, JEROME SEYMOUR (1969) Urna Nova Teoría da Aprendizagem. Rio de Janeiro: Bloch.

Capella y colaboradores (2003). Estilos de Aprendizaje en los Estudiantes de la Pontificia Universidad Católica del Perú. Lima: Pontificia Universidad Católica del Perú.

CASTRO, I. (2005). Hábitos de estudio y rendimiento académico de los alumnos del Instituto Superior Pedagógico Privado "Uriel García” del Cusco - 2005. Tesis de maestría. Lima: Universidad Cayetano Heredia.

JARA QUISPE, GLADYS ROSALÍA (2010) Tesis de maestría "Estilos de aprendizaje y rendimiento académico de estudiantes de 2 do de secundaria en educación para el trabajo de una Institución educativa del Callao. Lima-Perú: Universidad san Ignacio de Loyola.

MONEREO FONT, C. (2000) Estrategias de Aprendizaje. Madrid: Visor.

ORELLANA, O. (2003). Enseñanza aprendizaje y la mediación constructivista. Lima-Perú: San Marcos.

PIAGET, J. Y OTROS (1986): La enseñanza de las matemáticas modernas. Madrid. Alianza editorial.

POZO, J. I. Y MONEREO, C. (2000): EI aprendizaje estratégico. Madrid: Aula XXI Santillana.

VILDOSO V. (2003). Influencia de los hábitos de estudio y la autoestima en el rendimiento académico de los estudiantes de la escuela profesional de agronomía de la Universidad Nacional Jorge Basadre Grohmann. Tesis de maestría. Lima-Perú: Universidad Nacional Mayor de San Marcos. 